\title{
Geophysical Estimation of Permeability in Sedimentary Media with Porosities from 0 to $50 \%$
}

\author{
Jesús Díaz-Curiel ${ }^{1 *}$, Bárbara Biosca ${ }^{7}$ and María Jesús Miguel ${ }^{2}$ \\ ${ }^{1}$ Department of Geological Engineering, School of Mines, Universidad Politécnica de Madrid, C/Ríos Rosas 21, 28003 Madrid - Spain \\ 2 Spanish Ministry of Economy and Competitiveness, C/Albacete 5, 28071 Madrid - Spain \\ e-mail: j.diazcuriel@upm.es - barbara.biosca@upm.es - mjesus.miguel@mineco.es \\ * Corresponding author
}

\begin{abstract}
The main objective of this study is to find a relationship between permeability and porosity, in the range 0 up to 50\%, so that the permeability could be estimated only through geophysical parameters. This relationship should be consistent with the statistically expected values in sedimentary basins, and it should include the positive correlation between permeability and porosity in consolidated media and the negative correlation in unconsolidated ones.

We have also developed another relationship between formation factor and permeability, considering that the values of porosity and pore fluid resistivity are known from other sources different from resistivity logs. - The method for determining these relationships has been to generate functions with the expected geometry and to fit them to empirical data drawn from published papers. As a result we obtained a new relationship between porosity and permeability, with cementation exponent as added variable, and a new relationship for indirect estimation of permeability from the formation factor.

Given the formation factor dependence with clay content, we have also developed a theoretical relationship to calculate the formation factor in a clayey media, assuming that clay content is known from other logs. In this relationship, we have not taken into account the presence of non-conductive liquid phases, such as oil.

Comparison of the results obtained from these expressions with data collected in the literature leads to conclude that it is possible to determine the permeability of each layer in any zone from resistivity logs, if both the clay content and the resistivity of the interstitial fluid are known.
\end{abstract}

Résumé - Estimation géophysique de la perméabilité dans les milieux sédimentaires avec des porosités comprises entre 0 et $50 \%$ - L'objectif principal de cette étude est de trouver une relation entre la perméabilité et la porosité dans la gamme de 0 à $50 \%$, de sorte que la perméabilité puisse être estimée seulement par des paramètres géophysiques. Cette relation doit être statistiquement compatible avec les valeurs attendues dans les bassins sédimentaires, et inclure la corrélation positive entre la perméabilité et la porosité dans les milieux consolidés et la corrélation négative dans ceux non consolidés.

Nous avons également développé une autre relation entre le facteur de formation et la perméabilité, étant donné que les valeurs de la porosité et de la résistivité du fluide des pores sont connues de diverses sources autres que les diagraphies de résistivité. La méthode de détermination de ces relations a été générée en fonction de la géométrie attendue, et ajustée aux données empiriques des documents publiés. En conséquence, nous avons obtenu une nouvelle relation entre la porosité et la 
perméabilité, en utilisant l'exposant de cimentation comme une variable supplémentaire, et un nouveau rapport à l'estimation indirecte de la perméabilité à partir du facteur de formation.

Compte tenu de la dépendance du facteur de formation avec la teneur en argile, nous avons également mis au point une relation théorique pour calculer le facteur de formation à partir d'un support d'argile, en supposant que la teneur en argile est connue à partir d'autre diagraphie. À cet égard, nous n'avons pas tenu compte de la présence de phases liquides non conductrices, telles que l'huile.

La comparaison des résultats obtenus à partir de ces expressions avec des données collectées dans la littérature amène à conclure qu'il est possible de déterminer la perméabilité de chaque couche dans une zone des diagraphies de résistivité, si à la fois la teneur en argile et la résistivité du liquide interstitiel sont connues.

\section{NOMENCLATURE}

\begin{tabular}{|c|c|}
\hline$C$ & Conductance \\
\hline$F$ & Formation factor \\
\hline$\digamma a$ & Apparent formation factor \\
\hline$k$ & $\begin{array}{l}\text { Permeability (the ease of a fluid to flow through a } \\
\text { medium) }\end{array}$ \\
\hline$R$ & Resistance \\
\hline$S_{E}$ & Specific surface \\
\hline$V s h$ & Shale (or clay) content (fraction) \\
\hline$V_{C L A Y}$ & Clay volume \\
\hline$m$ & Cementation exponent \\
\hline$d_{g r}$ & Average grain diameter \\
\hline$\varnothing$ & $\begin{array}{l}\text { Intergranular porosity (the ratio of the pore vol- } \\
\text { ume to the total volume) }\end{array}$ \\
\hline$\rho$ & Resistivity \\
\hline$\rho_{0}$ & Rock resistivity (in Archie’s first law) \\
\hline$\rho_{B R}$ & Rock resistivity without non conducting phases \\
\hline$\rho_{C}$ & Clay resistivity \\
\hline$\rho_{C R}$ & Resistivity of the rock fraction filled with clay \\
\hline$\rho_{W}$ & Resistivity of the formation fluid \\
\hline$\rho_{W R}$ & Resistivity of the rock fraction filled with fluid \\
\hline$\rho_{W R 0}$ & Rock resistivity when $\sigma_{W} \rightarrow 0$ \\
\hline$\sigma$ & Conductivity \\
\hline$\sigma_{W}$ & Fluid conductivity \\
\hline$\sigma_{B R}$ & Rock conductivity without non conducting phases \\
\hline$\sigma_{B R 0}$ & Rock conductivity when $\sigma_{W} \rightarrow 0$ \\
\hline
\end{tabular}

\section{INTRODUCTION}

The permeability $(k)$ of rocks is one of the most relevant physical properties to characterization and management in the petroleum and groundwater industries. The estimation of permeability from well logs is of unquestionable interest because they provide information of all layers traversed by the borehole, in real environmental conditions at borehole scale. Moreover, well logging is relatively quick and inexpensive when compared to test tools such as repeat-formation-tests or drill-stem-tests, especially if standard logs such as resistivity and gamma ray (also available by logging-while-drilling or by using measurementswhile-drilling) are used. In fact, the current use of well logging in the petroleum industry demonstrates its utility, even taking into account the possible uncertainty of permeability estimation. That interest is heightened by the commercial transactions of exploitation rights, which are largely based on information contained in well logs.

Obtaining an expression that establishes a unique $k(\varnothing)$ relationship for any sedimentary environment is, in general, a difficult goal to achieve because there are many features (structure and compaction, grain size, sorting, grain shape, etc.) that affect this relationship. Moreover, it is a common thought that this goal is not possible. This aim is especially ambitious if it attempts to obtain an expression which is valid in media with presence of cement and/or clay.

Studies to estimate the permeability value through porosity have considered two different factors for sedimentary environments: the internal geometric features, such as specific surface, hydraulic radius, tortuosity, grain shape or packing and, on the other hand, the clay or shale content.

Over time, different techniques have been developed to estimate permeability. These techniques began with the use of granulometric data (when they were available) to which the porosity values (Ø) were added, since those are easier to obtain indirectly. In most works, the relationship for permeability estimation maintains an "effective" grain diameter, either with similar expressions to Kozeny's (Kozeny, 1927) or with power functions of porosity (Morrow et al., 1969; Bourbie and Zinszner, 1985; Revil and Cathles, 1999; Bernabé et al., 2003).

Moreover, as the above procedures did not achieve a widespread permeability estimation, other factors were added, such as tortuosity or water saturation. New theories were also developed, such as percolation, network statistics and fractal models. However, as in the case of effective diameter, the use of a set of empirical coefficients is required, because they are approaches of a mean behaviour for the set 
of layers of the different formations in a specific area. Both through the initial parameters, as well as through these other factors, permeability-porosity relationships used different coefficients for different areas, providing very different results of permeability for the same porosity.

The last challenge to predict permeability is focused on 3D imaging of the pore structure and reconstructing the diagenetic evolution, but again these processes required the knowledge of added factors such as the mineral growth not previously known. We consider that it is possible to obtain a $k(\varnothing)$ relationship regardless the rock type and diagenetic history assuming that they generate different geophysical responses.

Many of the developments cited for estimating permeability approach the problem from the knowledge of a rather long series of small-scale features. These processes made the estimation increasingly complex, since the obtained relationships required the use of different parameters and more specific experimental coefficients for each geological environment. In this sense, we should note that many of these factors are undefined for disturbed samples.

For this reason, the central aim of this communication was the estimation of permeability from the most common geophysical parameters and by using the smallest possible number of them.

To achieve this purpose, since there was not a single $k(\varnothing)$ for any sedimentary media, first we proceeded assuming that the formation factor $F$ (ratio between rock resistivity and pore fluid resistivity) is the parameter which maintains a more univocal relationship with the permeability.

Although in early works on permeability estimation, a good correlation between $k$ and $F$ data could be appreciated (Archie, 1942; Winsauer et al., 1952), this correlation was not expressed in a relationship form. Many authors have stated that formation factor maintains a narrow correlation with permeability (Schopper, 1966; Carothers, 1968; Sawyer et al., 1971; Ogbe and Bassiouni, 1978; Paterson, 1983; Wong et al., 1984; Purvance and Andricevic, 2000). These correlations did not attain a widespread use for any consolidated formation, which is partly due to computation of the formation factor focused on finding a single value for the cementation exponent for all layers of a specific geological formation. This paper also enhances this consideration, stating that the cementation exponent is not only different for each formation, but also it should be obtained for each analyzed layer.

Another reason why a $k(F)$ relationship was not further developed is, in our opinion, because the employed formation factor was not independent on clay content. The definition of formation factor is not directly applicable in the presence of clay because when other conductive elements are present, the ratio between the resistivity of the rock and the fluid does not have a constant value for different fluid conductivities. For this reason, traditionally the forma- tion factor is determined by non-conventional core testing (special core analysis) which, aside from scale considerations, involves a very high cost.

For the previously mentioned reasons, we have developed a new expression for $F$ that removes the influence of clay presence within formations. We have used an electrical analogy similar to that employed by Waxman and Smits (1968), although the methodology that we carry out differs from their approach in critical aspects. The expressions we have obtained required a theoretical development whose results do not match exactly with the Waxman and Smits ones, although show the same behaviour.

Regarding the appropriate formation factor, given that $F$ depends on the resistivity of the formation fluid, in layers partially filled with oil (considering that oil is not conductive) it should be necessary to include water saturation $\left(S_{W}\right)$ of pore fluid. We think that this inclusion should not be a complex step following a similar method as Archie's second law, but given the length of this work we have not considered this presence.

The next step of our methodology was to develop a $k(F)$ relationship that fits to a wide-ranging bibliographic data set. Just like in the measurements on samples, the new expression for $F$ requires the knowledge of interstitial fluid resistivities $\left(\rho_{W}\right)$, which does not change the goal of this work since the fluid resistivities could also be obtained from geophysical data, like for example spontaneous potential log. Ever since the Schlumberger brothers and Leonardon (1934) launched the spontaneous potential log, there have been many researchers who have used this possibility (Mounce and Rust, 1944; Doll, 1949; Wyllie, 1949; De Lima et al., 2005; Salazar et al., 2008).

As a third step, in order to obtain a $k(\varnothing)$ relationship, we replaced $F$ given by Archie's first law (Archie, 1942), $F=1 / \varnothing^{m}$, in the above $k(F)$ relationship. For different $(k, \varnothing)$ points, the relationship between them will be fixed by the values of the cementation exponent.

Finally, to obtain the definitive coefficients of $k(F)$ and $k(\varnothing)$ relationships, we took into account that the resulting $k(F)$ must fit to the compiled $(k, F)$ data, and simultaneously the resulting $k(\varnothing)$ must embrace the wide set of bibliographic $(k, \varnothing)$ data. Given the diversity of the data used, we have not focused our study on data that reach the greatest concordance with our hypothesis, but on the average behaviour for the set. This work does not intend to find exact laws, but to reach sufficiently valid expressions, non-existent to date, so that future researches may possibly be able to derive theoretical expressions from the general equations of physics.

Therefore, our objectives were to determine relationships between the formation factor and permeability, and between porosity and permeability, for porosities from 0 to $50 \%$, including both consolidated and unconsolidated media, not in a geological formation as a whole, but in each layer of 
any formation. We have tried to build these relationships so that they reflect the more general behaviour of permeabilityporosity in nature, and that would lead the scientific community to accept their reliability. Despite these main objectives, we have considered that showing the complete geometry of the permeability-porosity relationship for the full range of sedimentary media is an achievement in itself.

\section{BACKGROUND}

Flow of a fluid through a porous medium is characterized by the hydraulic conductivity, which considers the intrinsic permeability $k$ of the medium and, on the other hand, the density, viscosity and temperature of the fluid. The most complex parameter to determine is permeability, which has been the subject of numerous studies, as much in hydrogeology as in oil exploration.

\subsection{Permeability Estimation from Porosity}

Permeability estimation from geophysical techniques started with the measurement of parameters related to formation porosity. These parameters were mainly electrical resistivity, scattering and absorption of Gamma-Rays (density log), scattering and absorption of neutrons (neutron log), elastic waves propagation speed (mono-frequency sonic log or simply sonic $\log$ ), and lastly nuclear magnetic resonance; this last parameter is the only one from which it is said to provide direct information on permeability.

Regarding the relationships between the geometrical characteristics and the permeability, although some authors developed earlier expressions for the permeability as a function of grain size and porosity (Slichter, 1899; Uren, 1925), the most accepted relationships are those established by Kozeny (1927) and Carman (1937) shown in the following equations:

$$
k_{K C}=\frac{196}{S_{E}^{2}} \frac{\phi^{3}}{(1-\phi)^{2}}
$$

$S_{E}$ being the specific surface of grains. For spherical grains $S_{E}=6 / d_{g r}$, where $d_{g r}$ is the average grain diameter, so that Equation (1a) takes the form of Equation (1b):

$$
k_{K C}=5.45 \cdot d_{g r}^{2} \frac{\phi^{3}}{(1-\phi)^{2}}
$$

This expression provides permeability values in units of surface, which gives rise to the commonly used unit for permeability $\left(\mu \mathrm{m}^{2} \approx 1\right.$ Darcy) in oil.

The generality of the Kozeny relationship (Eq. 1b) arises from the fact that it is possible to consider different porosities and different effective surfaces for the pores of a medium (different "effective" grain diameters). Despite its widespread application, the influence of grain size in the Kozeny equation does not consider the sorting as it was proposed by Berg (1970), who used a percentile of granulometric deviation, or van Baaren (1979), who included a sorting coefficient which varies between 0.7 and 1.0. The inclusion of sorting improves the indirect estimation of permeability, but it still omits packing so as to complete the geometric parameters information. To date, however, it is a widespread opinion that relationships for permeability estimation from porosity values are only considered adequate for each specific zone.

\subsection{Porosity-Permeability Correlation in Sedimentary Media}

In order to establish a $k(\varnothing)$ relationship for the entire range of porosities from 0 to $50 \%$, it is appropriate to analyze a particular aspect of the correlation that occurs between porosity and permeability in sedimentary environments. In a large number of studies on oil drilling, the existence of a positive correlation between $k$ and $\varnothing$ is identified (Archie, 1942; Winsauer et al., 1952; Carothers, 1968; Timur, 1968; Waxman and Smits, 1968; Turner, 1983; Sen et al., 1990; Nelson, 1994; Pape et al., 1999; Ehrenberg et al., 2006; Glover and Walker, 2009).

However, in studies of well data in non-consolidated media, this correlation is found to be negative (Jones and Buford, 1951; Baker et al., 1964; Alger, 1966; Croft, 1971; Worthington, 1977; Heigold et al., 1979; Urish, 1981; Worthington, 1983; Kwader, 1985; Kelly and Frohlich, 1985; Huntley, 1986; Detmer, 1995; Díaz-Curiel, 1995; Frohlich et al., 1996; Purvance and Andricevic, 2000) and others (Khalil and Santos, 2009). Some of these studies concluded by setting relationships for $k(\varnothing)$, usually approximated to linear or power functions. We should note that in these studies, the existence of this negative correlation was verified, although in some of them the data processing was not as comprehensive as in studies concerning oil.

\subsection{Porosity and Formation Factor}

After Sundberg (1932) established that the relationship between resistivity formation $\rho_{0}$ and the resistivity $\rho_{W}$ of the fluid that fills the pores had a constant value, Archie (1942) stated that the formation resistivity factor $F$, defined as the ratio of the resistivity $\rho_{0}$ of a formation completely saturated with a fluid of resistivity $\rho_{W}$ and such resistivity, is inversely proportional to a power of the porosity value, that is:

$$
F=\frac{\rho_{0}}{\rho_{W}}=\frac{1}{\phi^{m}}
$$


where " $m$ " is an exponent which, although Archie (1942) did not name it in any way, is called the cementation exponent. Equation (2) is known as Archie's first law.

Many studies seeking to determine formation factor went on with Archie's first law. In particular, Winsauer et al. (1952) added a factor " $a$ " to the formation factor expression, obtaining the relationship $F=a / \emptyset^{m}$, for which they found the values $a=0.62$ and $m=2.15$ to fit the experimental data. Following the proposal of Winsauer et al. (1952), other authors such as Carothers (1968), Porter and Carothers (1970) or Timur (1968), studied the best combinations for " $a$ " and " $m$ " which represent the average behavior of different formations. In this paper, we have assumed that the best relationship for the formation factor of sands without clay (or shale) is Archie's first law, i.e., excluding the " $a$ " coefficient, because, although this factor yields better fittings, it does not comply with $F$ equal to 1 if the porosity is $100 \%$ (all the medium is fluid).

This use of Archie's first law was continued by other authors who found a relationship between $F$ and porosity of all the porous layers at each borehole, and even in each area obtaining the slope of the regression line in the graph $\log (\digamma)$ versus $\log (\varnothing)$, (Jackson et al., 1978; Salem and Chilingarian, 1999; Glover and Walker, 2009). As we have said in the introduction section, we consider that this is only appropriate for a specific geological formation, while in general the cementation exponent should be obtained for each analyzed layer.

\subsection{Formation Factor in Clayey Media}

Archie's first law was established for clean sands, for which both the measured and the real formation factors coincide. However in clayey media this does not occur because the clay content involves simultaneously a permeability reduction and a decrease in the resistivity of the medium, as it adds electrical conductivity (superficial and intrinsic).

The study of the influence of clay content in geophysical parameters began with the work of Patnode and Wyllie (1950), followed by De Witte (1950), Winsauer and McCardell (1953), Hill and Milburn (1956), and Waxman and Smits (1968). These works concluded that when there are conductive materials between grains, the obtained factor is an apparent formation factor, and an analogy with electrical circuits was adopted to calculate the formation factor of clayey or shaly formations.

Waxman and Smits (1968) assumed that $F$ is the same for the fluid and for the clay, and they considered that the electrical resistance is the parameter that maintains the analogy with electrical circuits. So, for elements in parallel, the conductance of the set is the sum of the conductances (in their
Equation (1): $C_{r o c k}=C_{c}+C_{e l}$, where $C_{r o c k}, C_{c}$, and $C_{e l}$ are the conductances of rock, of exchange cations associated with clay, and of free electrolyte, respectively). Then, in their Equation (2), they stated that $\sigma_{0}=x \cdot \sigma_{e}+y \cdot \sigma_{w}$, where $\sigma_{0}, \sigma_{e}$ and $\sigma_{w}$ are the conductivities of core, of clay exchange cations, and of salt solution in equilibrium, respectively; and $x$ and $y$ are the appropriate geometric constants. After, they assumed that the geometric constant is the inverse of the formation factor (according to their Equation (3) $\left(x=y=1 / F^{*}\right)$. Finally, the main drawback of this work was expressed in their Equation (4) $\left(\sigma_{0}=\left[\sigma_{e}+\sigma_{w}\right] / F^{*}\right)$.

In the literature, one can find different arguments that limit the use of Archie's first law to obtain the formation factor when conductive compounds such as clay are present. Although some authors consider that Archie's first law is not applicable in the presence of clays (De Lima and Sharma, 1990; Jin and Sharma, 1994; Herrick and Kennedy, 1995), we have taken up again the cited developments quoted above.

\section{METHODOLOGY}

As stated by Sen et al. (1990) "Empirical laws have been extremely useful in geophysical exploration". In the case of the $k(\varnothing)$ relationship, it has been very common to find an expression that presents the minimum error against empirical data from one or more zones with similar characteristics. In order to use it in any sedimentary medium, and to allow the estimation of the permeability of each layer from geophysical parameters, we have searched for fitting functions that adapt to the general behaviour, instead of pursuing the minimum error.

The mathematical expression of the chosen fitting function, although it is not among the best known conventional regression functions (power, polynomial, exponential or logarithmic), does not involve any theoretical deduction but simply a knowledge of the geometry of certain mathematical expressions that are similar to that shown by permeability data versus formation factor and versus porosity.

Figure 1 shows the $(k, \varnothing)$ data of some of the works cited in Section 1.2. Relating to this data, we must mention that some of them have been extracted directly from the papers and other data have been extracted from figures. We have established some bands that embrace the compiled data, as general ranges of $(k, \varnothing)$ relations for the two behaviours, positive and negative. These bands are simply drawing curves, without any mathematical expression, in order to define the geometry and the confined space of the functions we must develop. We must point out that most of published data are located inside the space covered by these band, therefore this space can be considered enough widespread for all sedimentary media. 


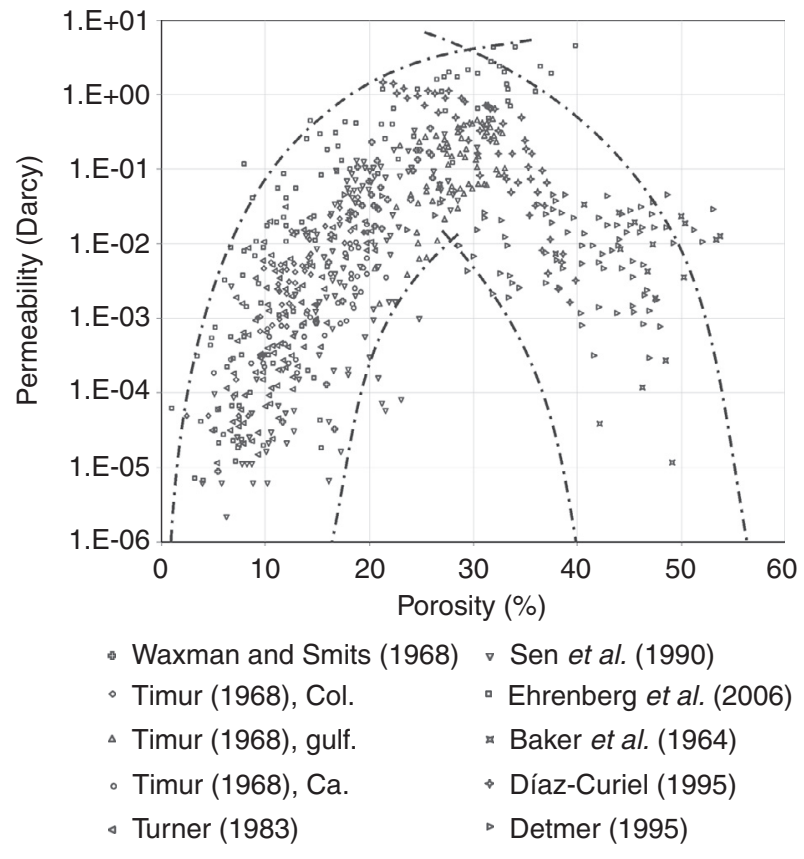

Figure 1

$k$, Ø values, for consolidated and unconsolidated media, extracted from the literature, with drawing of proposed bands (dashed line) that embrace them.

The points spread in the $(k, \varnothing)$ space led us to think of an adjustment to a beta distribution, $k=(A \cdot \varnothing)^{B}(1-\varnothing)^{C}$ as the first option, but there was a wide variety of combinations for these three coefficients. Although the best-fit values were $A=25.7 / \mathrm{m}^{1.70}, B=10.6 \cdot \mathrm{m}^{1.57}$, and $C=86.9 \cdot \mathrm{m}^{0.27}$, their determination was somewhat unjustified.

For this reason, starting from this general range of porosity-permeability values, in order to develop a relationship that linked them, we have simultaneously adopted and solved several assumptions and premises, which are interconnected, between porosity and permeability:

1) electrical porosity is in some way related to ionic mobility, and therefore to permeability. This is a correspondence that is widely accepted;

2) it is possible to group into a single mathematical expression the geometric characteristics which are independent of the presence of clay, both for high and low porosities. For high porosity values, this expression considers the reduction of grain size and for low values it also considers the presence of cement, so that, in practice, both factors have an influence on permeability values, although in different proportions;

3 ) it is possible to develop a theoretical expression of the formation factor, which removes the effect of the presence of clays in its calculation, based on measurable well $\log$ values;

4) the formation factor maintains a straightforward relationship with the permeability, because it has an implicit relationship, through Archie's first law, with both the porosity and the cementation exponent;

5) the $k(\varnothing)$ relationship that occurs during the sedimentation process can be fitted by a function that considers the equality of permeability values for those media that have the same formation factor.

The first two assumptions are general hypotheses and do not involve a specific development, therefore we present the development of the latter three below.

\subsection{Development of Hypothesis 3}

As we mentioned above, all premises and hypotheses are interrelated, especially the $k(\varnothing)$ and $k(F)$ relationships. However, the $k(\varnothing)$ and $k(F)$ relationships that we were looking for are highly influenced by the presence of clays, with its consequent increase in the electrical conduction.

To remove this influence in the formation factor, we assumed (Waxman and Smits, 1968) that $F$ is the same for the fluid and for the clay, at least for percentages of clay content below $50 \%$. In order to consider clay conduction numerically, we have also considered as in Waxman and Smits, that the electrical resistance, not the resistivity, is the parameter that maintains the analogy with electrical circuits.

However, we disagree with their subsequent formulation. First, in their Equation (2), the geometric constant should have also been applied to the core, otherwise its conductance would be the same as its conductivity. Second, we think that the assumption that the geometric constant is the inverse of the formation factor in their Equation (3) is not correct; if it were right, the formation factor of, for example, a cylindrical sample would be different when its length was different, which is not true. Finally, we think that the main drawback of Equation (4) of Waxman and Smits (1968) (the final key on its electrical analogy) is that the fraction of each conductive components does not appear. The fractions of the rock filled with fluid and filled with clay should affect the rock resistivity as a function of their ratios, since otherwise the rock resistivity would be the same for any ratio.

In our model, the electrical resistance of a rock sample composed of different conductive elements is obtained by adding conductances of the elements that are distributed transversely to the current and by adding resistances for those distributed longitudinally to the current. As any previous established configuration, we considered isotropic samples, that is, with a resistance with the same proportion transversally and longitudinally. Then, the electrical analogy that we employ consists of a circuit composed by $N_{1}$ resistances $R_{1}$ in parallel with $N_{2}$ resistances $R_{2}$. 
The resistance of each in-serial set, $R_{E S}$, will be:

$$
\frac{1}{R_{E S}}=\frac{N_{1}}{R_{1}}+\frac{N_{2}}{R_{2}} \Rightarrow R_{E S}=\frac{R_{1} \cdot R_{2}}{N_{1} \cdot R_{2}+N_{2} \cdot R_{1}}
$$

If we consider a gathering of $M$ in-serial sets, the total resistance will be the sum of their resistances, that is, $R_{T O T}=M \cdot R_{E S}$, then multiplying by $\left(N_{1}+N_{2}\right)$ in both sides of the former equation, and inverting:

$$
\frac{1}{R_{T O T}} \frac{M}{\left(N_{1}+N_{2}\right)}=\frac{N_{1} /\left(N_{1}+N_{2}\right)}{R_{1}}+\frac{N_{2} /\left(N_{1}+N_{2}\right)}{R_{2}}
$$

where $N_{J} /\left(N_{1}+N_{2}\right)$ provides the fraction of resistances $R_{J}$ in the sum of resistances.

To continue the analogy, let us consider a rock sample composed of $\left(N_{1}+N_{2}\right) \cdot M$ volume elements, $N_{1}$ of resistance $R_{1}$ filled with a conductive material, and $N_{2}$ of resistance $R_{2}$ filled with another conductive material. Let us also consider the volume of each element, $\Delta V=\Delta S \cdot \Delta L$, in such a way that the length sample $L=M \cdot \Delta L$ and the cross section $S=\left(N_{1}+N_{2}\right) \cdot \Delta S$. Naming $\rho_{1}$ and $\rho_{2}$ the resistivity of the elements 1 and 2 respectively, and $\rho_{B R}$ the resistivity of the rock as a whole, the resistance of each element will be given by $R_{J}=\rho_{J} \cdot \Delta L / \Delta S$, and of the sample by $R_{T O T}=\rho_{B R} \cdot L / S$, then:

$$
\frac{1}{\rho_{B R}}=\frac{N_{1} /\left(N_{1}+N_{2}\right)}{\rho_{1}}+\frac{N_{2} /\left(N_{1}+N_{2}\right)}{\rho_{2}}
$$

We should note that we use the subscript ' $B R$ ' rather than the conventional subscript ' $T$ ' that is usually employed for true resistivity, because we have not considered the presence of non-conductive liquid phases, such as oil.

Let us suppose a rock with clay or shale content $V s h=V_{C L A Y} / V_{T O T A L}$, and porosity $\varnothing=V_{H O L E} / V_{T O T A L}$; dividing both expressions, the pore fraction filled with clay $V_{C L A Y} / V_{H O L E}=V S h / \varnothing$ is obtained. The other fraction of the pores, those filled with fluid would be given by:

$$
1-V s h / \varnothing=(\varnothing-V s h) / \varnothing
$$

Continuing the electrical analogy, if we consider that "1" elements correspond to the portions of the sample filled with fluid and " 2 " to those filled with clay, Equation (3b) becomes:

$$
\frac{1}{\rho_{B R}}=\frac{(\phi-V s h) / \phi}{\rho_{W R}}+\frac{V s h / \phi}{\rho_{C R}}
$$

where $\rho_{W R}$ and $\rho_{C R}$ stand for the resistivities of the rock fraction filled with fluid and the rock fraction filled with clay, respectively.
Every part of the rock, the one filled with fluid and the one filled with clay, will keep a proportional relationship between its resistivity and the resistivity of its filling given by the formation factor, then:

$$
\frac{1}{\rho_{B R}}=\frac{(\phi-V s h) / \phi}{F \cdot \rho_{\mathrm{W}}}+\frac{V s h / \phi}{F \cdot \rho_{\mathrm{C}}}
$$

where $\rho_{W}$ and $\rho_{C}$ represent the resistivities of the fluid and clay, respectively.

From Equation (3d), the formation factor is obtained as a function of clay content by the following relationship:

$$
F=\rho_{B R}\left(\frac{(\phi-V s h) / \phi}{\rho_{W}}+\frac{V s h / \phi}{\rho_{C}}\right)
$$

If one expresses Equation (3d), in a straight line form:

$$
\frac{1}{\rho_{B R}}=\frac{1}{\rho_{W R 0}}+\frac{1}{F a} \frac{1}{\rho_{W}}
$$

where:

$$
\frac{1}{\rho_{W R 0}}=\frac{V s h / \phi}{F \cdot \rho_{C}}
$$

and

$$
\frac{1}{F_{a}}=\frac{(\phi-V s h) / \phi}{F}
$$

$F_{a}$ being an apparent formation factor (the slope of rock conductivity $\sigma_{B R}$ versus fluid conductivity $\sigma_{W}$ ).

Equation (3f) shows a similar behaviour than the classical $\sigma_{B R}\left(\sigma_{W}\right)$ one. For zero values of clay content, Equation (3f) turns out to be the conventional expression for the formation factor, and if $V s h=\varnothing, \sigma_{B R}$ is constant for any fluid conductivity. The most important difference is that the slope of $\sigma_{B R}\left(\sigma_{W}\right)$ is different and is function of the ratio $(\varnothing-V s h) / \varnothing$.

\subsection{Development of Hypothesis 4}

As we have mentioned in the Introduction and Background sections, the formation factor is one of the most analogous geophysical parameters to the permeability because it reflects the easiness of the movement of ions present in a conductive fluid through a porous medium, independent of the fluid conductivity (ionic content).

Data on the positive correlation between $k$ and $F$ for consolidated sands was reflected in the $(k, F)$ graphs presented by Archie (1942), Winsauer et al. (1952), Waxman and Smits (1968), Carothers (1968), Sawyer et al. (1971), and Sen et al. (1990), among others. Similarly, for unconsolidated 


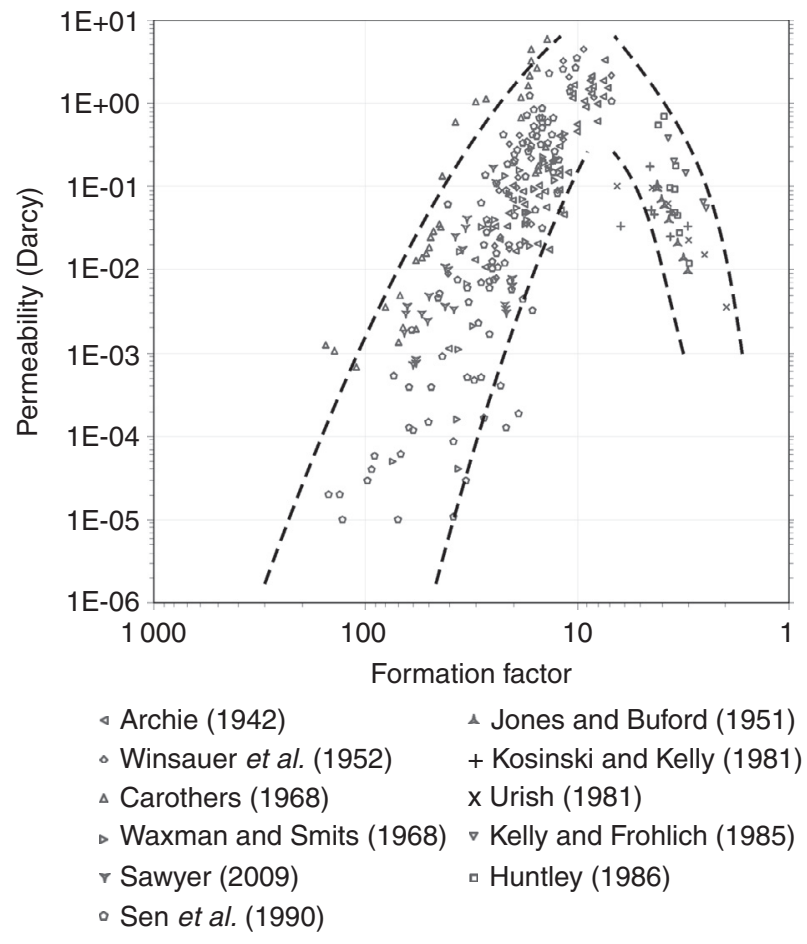

Figure 2

$k, F$ data for consolidated and unconsolidated media from bibliography, with the approximated bands that embrace them.

media there are also available data on the negative correlation between $k$ and $F$, of which we have selected those presented by Jones and Buford (1951), Kosinski and Kelly (1981), Urish (1981), Kelly and Frohlich (1985) and Huntley (1986).

Figure 2 shows data extracted from these works, in which we have not taken into account the very shaly sandstone data of Waxman and Smits (1968) and the limestone data from Carothers (1968). As in $(k, \varnothing)$ data, some data have been taken directly from the papers and others extracted from figures. We consider that selected works are representative of the general behaviour of $k$ versus $F$ in sedimentary media. We must point out that the two different performance of $k(F)$, the one for $F$ greater than $\sim 10$ and the other for $F$ lower than $\sim 10$, was already shown by Worthington (1983), although in this work a relationship to reflect this behaviour was not developed.

As in Figure 1, Figure 2 includes some bands that embrace the data cited above as general ranges of $(k, F)$ relations for the two behaviours, positive and negative; and again these bands are simply drawing lines, without any mathematical expression, in order to define the comprehensive geometry. So, the development of this hypothesis consisted of searching a function that relates the $(k, F)$ extracted data.
Besides achieving a good fit to the data, the $k(F)$ relationship should reflect the two mentioned behaviours that occur in sedimentary environments around a maximum value, that is, the decrease of permeability, due to the decrease of porosity in consolidated media when $F$ increases, and the increase of fluid retention in unconsolidated media when $F$ decreases. To sum up, permeability must increase with $F$ for low $F$ values $(2<F<\sim 10)$, and decrease for $F>\sim 10$. Based on these requirements we have decided to use the product of two functions that would tend to zero for values of $F=1$ and $F=\infty$, and which would produce the maximum value of permeability in the range of the formation factor between 5 and 10 , corresponding to clean sand or very well sorted gravel.

With these criteria, the selected product of functions was an increasing hyperbolic function, $1 / F^{f_{3}}$, multiplied by an algebraic decreasing function $(F-1)^{f_{2}}$, and both multiplied by a constant $f_{1}$, where $f_{1}, f_{2}$, and $f_{3}$ are the fitting coefficients. Then, the form of the relationship $k(F)$ is given by following equation:

$$
k=f_{1} \frac{(F-1)^{\mathrm{f}_{2}}}{F^{f_{3}}}
$$

Although the number of data values for permeability versus formation factor is much lower than the ones existing in the literature for $(k, \varnothing)$, they have a much greater dispersion than it would be expected according to the hypothesis of a unique relationship between $F$ and $k$. This led us to make a detailed analysis of them. The first fact we noted is that, in some works on hydrogeological boreholes, remoulded samples were used. On the other hand, studies both on data from unconsolidated samples and oil borehole data have used different procedures to calculate the formation factor than the one we are proposing in this work, which may explain this dispersion. In some cases, formation factors are obtained from regression lines, $\log (F)-\log (\varnothing)$, in core sets. This procedure considers a single cementation exponent for each set of samples, which is contrary to the assumptions of this work (a cementation exponent for each layer), and overestimates the formation factor, so the bands defined in Figure 2 do not include such data. Finally, we must note that, although the band is much narrower in non-consolidated media, there is also a considerable dispersion, because the variation range of the formation factor in these media is much smaller.

On the basis of the foregoing considerations, the method used to determine the optimal coefficients was to select those that achieve a better adjustment to the trends of the data presented in Figure 2, and not those that produce the minimum error with respect to data values; that is, these coefficients have been selected to produce the best correlation with the behaviour of the data. The relationship between permeability 


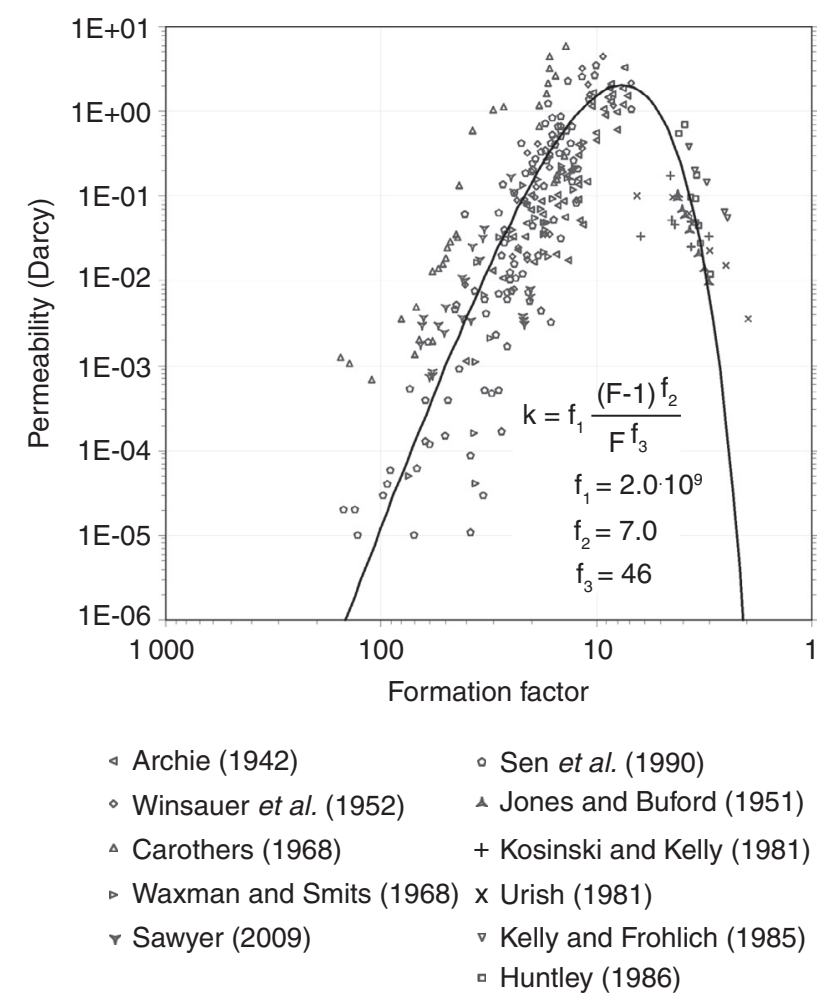

Figure 3

Permeability curve versus formation factor following Equation ( $4 \mathrm{~b})$, and $k, F$ data for consolidated and unconsolidated media from bibliography.

and the formation factor with the resulting coefficients are presented in the following equation, and their geometry is shown in Figure 3.

$$
k=2.0 \cdot 10^{9} \frac{(F-1)^{7}}{F^{46}}
$$

Equation (4b), of $k(F)$ is valid for formation factor values of $2.0<F<200$, which is a generally accepted range for sedimentary environments (Sundberg, 1932). In general, given that $F>2$ for most porous media, it is geologically pointless to pursue its validity in the limit of $F \rightarrow 1$, which according to Equation (4b), of $k(F)$ would correspond to zero permeability.

At this point, it should be mentioned that the data of some referenced works taken separately have a very marked alignment; for example $(k, F)$ data from Archie (1942), Winsauer et al. (1952), for consolidated media, and Jones and Buford (1951), Huntley (1986), or Kelly and Frohlich (1985) for unconsolidated media. This leads us to doubt whether the curve that we have adopted for the $k(F)$ relationship is based on optimal coefficients, as the change of the coefficients to adjust to a specific data set is a simple process. Certainly the sources of the used data are very diverse, and therefore it is not possible to assure that the measurements have been done in the same conditions.

We consider, for example, that the formation factors conventionally obtained, by calculating the slope in a graph $\log (F)-\log (\varnothing)$, may be very different from the actual values. Thus, for example, if the cementation exponents for a series of samples with low porosity ( 5 to $25 \%$ ), vary by six tenths (let us take as an example the range from 1.6 to 2.2), the obtained formation factors become five times greater than the actual values. Although this would lead us to adopt different coefficients, both in the interest of the generality of Equation (4b), of $k(F)$, as well as to avoid disagreements with published works, we have decided to take the coefficients resulting from fitting to all data.

In the same sense, it should be noted that the values of the obtained coefficients to adjust Equation (4a) to the $(k, F)$ data, have not been the initial ones. The coefficients were slightly modified so that they would also produce the best fitting to the trends of $(k, \varnothing)$ data, after completing the $k(\varnothing)$ equations that are described in the development of the following hypothesis.

\subsection{Development of Hypothesis 5}

Assuming that Archie's first law should be retained, once the $k(F)$ relationship is adopted, the $k(\varnothing)$ function was obtained by replacing $F$ by $1 / \oslash^{m}$. The resulting expression for the $k(\varnothing)$ relationship will be as follows:

$$
k=M_{1} \cdot \phi^{M_{2}}\left(1-\phi^{m}\right)^{M_{3}}
$$

which has a similar behaviour to a beta distribution as we supposed initially, that is, $M_{2}$ and $M_{3}$ are coefficients which determine the geometry in each case, while $M_{1}$ fixes the maximum value.

To obtain the coefficients of Equation (5a), of the $k(\varnothing)$ relationship, we have related them to the cementation exponent, forcing the $K(\varnothing)$ expression to comply simultaneously with two requirements. The resulting values must fit with those typical values found in former works, and provide the same permeability in those cases $(\varnothing, m)$, corresponding to the same formation factor (which matches with the link between formation factor and water mobility assumed in this work).

The first requirement restricts the range of values that $M_{2}$ and $M_{3}$ could take, while the second condition establishes the relationship between them, because if we consider two media with characteristics $\left(\varnothing_{p}, m_{p}\right)$ and $\left(\varnothing_{q}, m_{q}\right)$, and with the same formation factor, following Archie's first law, we will have:

$$
1 / \phi_{p}^{m_{p}}=1 / \phi_{q}^{m_{q}} \Rightarrow \phi_{p}^{m_{p}}=\phi_{q}^{m_{q}}
$$




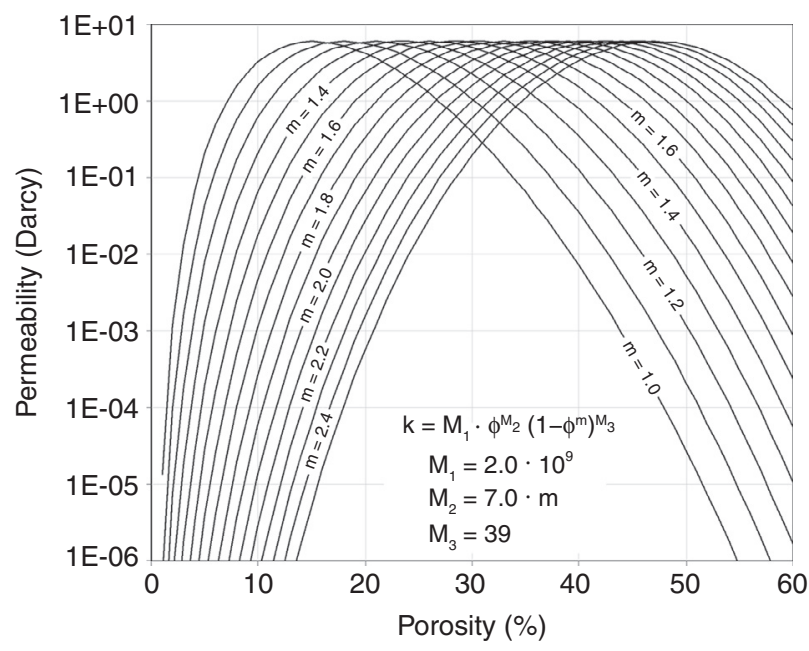

Figure 4

Permeability versus porosity curves according to Equation (5b) for different values of cementation exponent $m$, ranging between 1.0 and 2.5 .

This determines the relative position of both points on the curve, and implies that, regarding permeability behaviour, a given porosity value must be accompanied with a unique value of " $m$ " as a function of its formation factor.

Considering these requirements, the resulting relationship between permeability as a function of the porosity is presented below:

$$
k=2.0 \cdot 10^{9} \cdot \phi^{7 m} \cdot\left(1-\phi^{m}\right)^{39}
$$

where the value of the first constant matches Equation (4b), of $k(F)$. The $k(\varnothing)$ curves obtained using Equation (5b) of $k(\varnothing)$, for different values of the cementation exponent, are presented in Figure 4.

The greater absolute values of the slopes in Equation (5b) of $k(\varnothing)$ for consolidated media respecting unconsolidated ones, come to reflect the fact that decreasing of permeability in consolidated media occurs at a time with the cement presence. In any case, for consolidated media, the greater " $m$ " the lower $k$, while in unconsolidated media, the higher " $m$ " the higher $k$.

We must remark that Equation (5b) does not mean a single behaviour of $k(\varnothing)$ for all sedimentary media, rather on the contrary, only a specific set of data will have the shape of the curve for a given " $m$ " (those with the same cementation exponent). In general, each layer with a certain value of $\varnothing$ will have a unique value of permeability as a function of its " $m$ " value.

The range of cementation exponents for which Equation (5b), of $k(\varnothing)$ is deemed valid is found between $m>1.20$ and $m<2.40$. This range has been chosen because it is a middle range for sedimentary formations (Archie, 1942; Wyllie and Gregory, 1953; Coates and Dumanoir, 1974; Katz and Thompson, 1985; Ehrlich et al., 1991; Byrnes et al., 2009; Lee, 2011).

Since this range of variation of " $m$ ", is very small, the value $m=1.5$ is much higher, in relative terms, than $m=1.2$. Moreover, the values of the $k(\varnothing)$ function imply a high degree of variation of $k$ depending on the cementation exponent values. For these reasons we consider that this exponent should be presented with at least two significant digits.

\subsection{Validation of Results}

First of all, although the degree of uncertainty between the results of Equations (4) and (5) and the compiled data may be high, it is not opposite to the main objective of this work. We consider that it is functional to use a mean curve of the available data for a wide range of sedimentary media because otherwise it would imply that the data provided by each author are not valid. In fact, the difference among data from different studies will always be higher than the difference between each one of them and the mean curve.

In order to estimate the degree of uncertainty of Equation (4b) of $k(F)$, we have computed the mean difference between the compiled data and the predicted ones, resulting $<0.7$ orders of magnitude. These results may be considered as a good enough approximation for the identified goal of this work. We should note that the $(k, F)$ data are very dispersed, as the mean difference of all data in each branch (positive and negative) is 0.7 orders of magnitude with regard to the usual power functions.

Regarding the results of Equation (5b), firstly it should be noted that the $k(\varnothing)$ curves (Fig. 4) obtained in this paper provide values that are consistent with the different works performed to date for porosities less than $30 \%$.

To illustrate this, we have extracted data from the works of Timur (1968), Waxman and Smits (1968), Turner (1983) and Sen et al. (1990) and we have represented the $k(\varnothing)$ curves, given by Equation (5b), in Figure 5, together with the different cementation exponents that contain the compiled data.

As it is observed in Figure 5, data from these studies are included in the range of curves obtained from Equation (5b) of $k(\varnothing)$, and most of the data set match a particular curve, that is to say, with a certain cementation exponent. The cementation exponent values vary, for the set of those data, between 1.35 and 2.50, and these are quite reasonable if we consider that clay content has not been taken into account. The cementation exponent of the $k(\varnothing)$ expression that embraces the Waxman and Smits (1968) data is between 


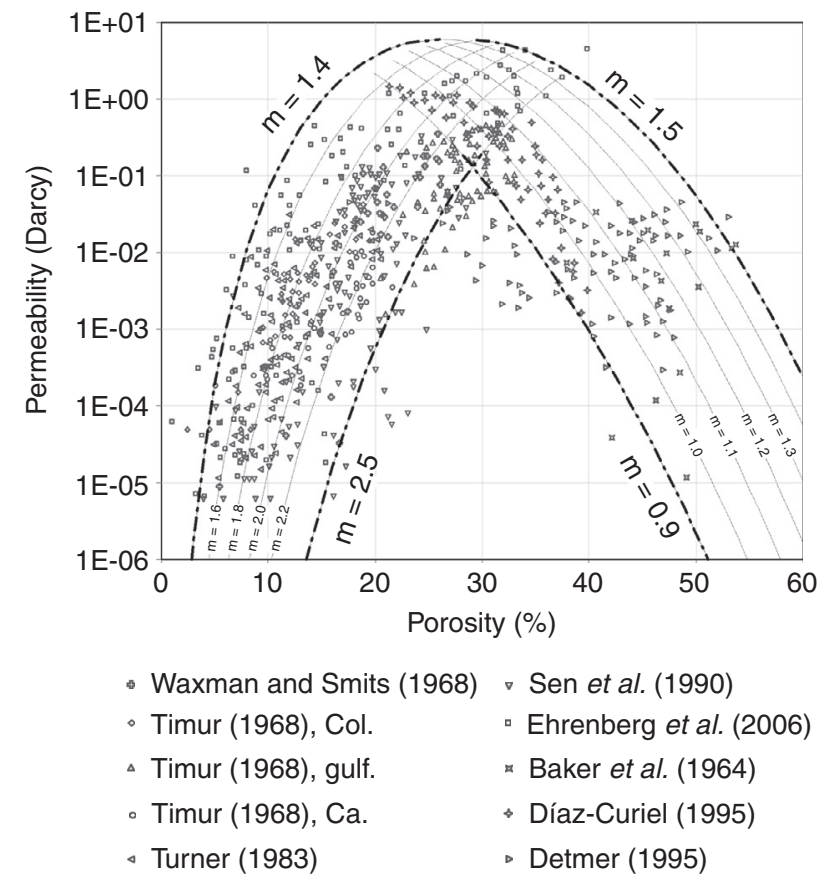

Figure 5

Permeability versus porosity referenced data, and permeability curves according to Equation (5b) of $k(\varnothing)$ for different $m$.

1.9 and 2.5 , for the $99 \%$ of the data from Turner (1983) is between 1.5 and 2.2, and for the $96 \%$ of Timur (1968) data from Colorado, is between 1.6 and 2.1.

Among the various comments on the different cases, it is worth mentioning the high correlation of the derived $k(\varnothing)$ curves with data submitted by Waxman and Smits (1968) and Turner (1983). The data from Timur (1968) in the Californian area exhibit a more horizontal distribution than the remaining data presented, and therefore some of them lie outside the stated range for that area. Finally, we have also decided to leave some of the data gathered by Sen et al. (1990) outside the established range; in particular, three cases of sandstones with very low porosity in comparison with their permeability value.

A similar gathering is shown in Figure 5 for unconsolidated media on extracted data from the work of Baker et al. (1964), Detmer (1995) and Díaz-Curiel (1995), together with the $k(\varnothing)$ curves given by Equation (5b) of $k(\varnothing)$ that contain them, with the corresponding cementation exponent.

We have represented the curves given by Equation (5b) for $K(\varnothing)$, for values of $m=0.9$ and $m=1.5$, leaving out of range data from the upper right, since they have permeability values of $\sim 0.1$ Darcy for porosities of about $50 \%$, which are fairly uncommon simultaneously. The range of " $m$ " values for Equation (5b), of $k(\varnothing)$, that set the limits for all Díaz-Curiel (1995) data are between 0.9 and 1.20, for the $90 \%$ of Baker et al. (1964) data between 0.95 and 1.5 , and for the $93 \%$ of Detmer (1995) data between 0.9 and 1.4.

In order to know a degree of uncertainty for Equation (5b) of $k(\varnothing)$, we have taken the $(k, \varnothing)$ values from Glover and Walker (2009). The mean difference with the $k(\varnothing)$ values obtained from Equation (5b) for $m=1.9$, is 0.3 order of magnitude, which once again we consider a sufficiently close approximation for the goal of this work. We have showed both together in Figure 6, in which it can be seen that the cementation exponent from Glover and Walker (2009) data for porosities $\geq 19 \%$ are smaller than the remainder.

We performed the same test for unconsolidated media on extracted data from the work of Díaz-Curiel (1995) and contrasted them with the $k(\varnothing)$ values obtained from Equation $(5 \mathrm{~b})$ for $m=1.0$ (Fig. 6), resulting in an uncertainty average of 0.31 order of magnitude. In this case, the highest cementation exponent takes place for porosities around $27-35 \%$.

In any case, we would note that the influence of both the precision in the cementation exponent and the values of the coefficient of the $k(F)$ relationship produce a high level of uncertainty that is added to well logs (work on advanced calculation of this uncertainty can be seen in Viberti and Verga, 2011).

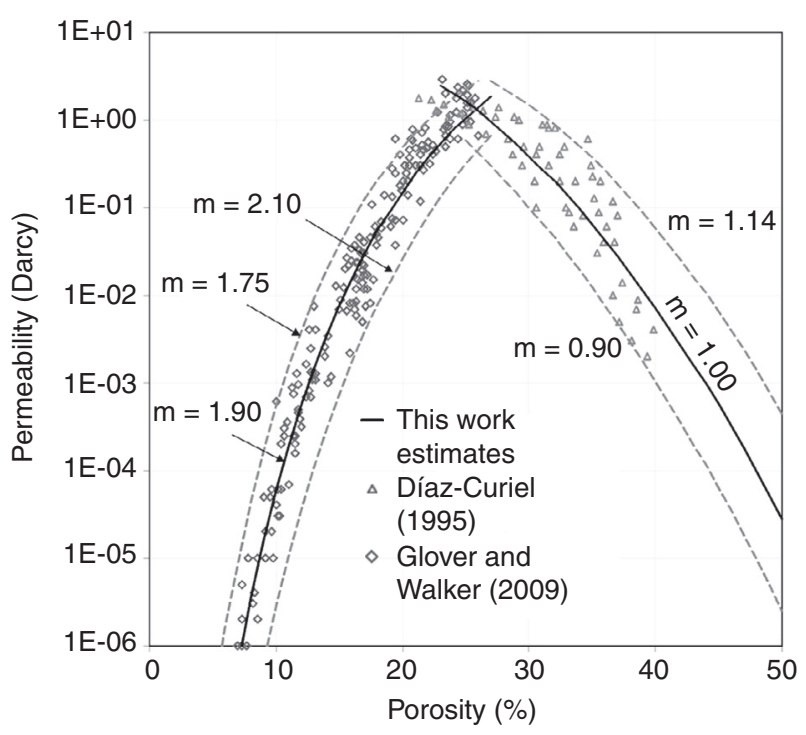

Figure 6

Permeability versus porosity data from Glover and Walker (2009) (diamonds) and from Díaz-Curiel (1995) (triangles). Average $k(F)$ obtained from Equation (5b) (solid line) and bands (dashed line) of the same equation that embraces the data. 


\section{DISCUSSION}

\subsection{About $\sigma_{B R}\left(\sigma_{W}\right)$ Behaviour of Equation (3d)}

In order to compare the behaviours of Equations (3) and the classical Waxman and Smits ones, let us consider an example with $\varnothing=30 \%, V_{s h}=10 \%$ (the pore fraction filled with clay is $33 \%$ ), $\rho_{C}=10 \Omega \cdot \mathrm{m}$, and $F=8$. Drawing (Fig. 7) the $\sigma_{B R}\left(\sigma_{W}\right)$ curve given by Equation (3f), and at the same time the apparent formation factor, $F_{a}=\sigma_{W} / \sigma_{B R}$, it is evident that the trend of the $\sigma_{B R}\left(\sigma_{W}\right)$ curve is not the same in the upper and lower limits of fluid conductivity.

When $\sigma_{W}$ tends to the maximum value (at ambient temperature), the rock conductivity shows a similar behaviour as that from rocks whose pores are filled only with fluid, namely proportional to $\sigma_{W}$. However, the apparent formation factor tends to a value of 12 , which would be considered as the real value in the Waxman and Smits model, contrary to the employed data in the example $(F=8)$. That value (12) derives from the fact that the rock is partially filled $(33 \%)$ with clay; therefore it will not present the same value as if the pores were filled only with fluid.

As it is verifiable throughout the literature since Waxman and Smits (1968) until Byrnes et al. (2009), when $\sigma_{B R}\left(\sigma_{W}\right)$ shows an increasing quasilinear behaviour in bilogarithmic scale, which implies that $F_{a}$ values increase with increasing $\sigma_{W}$. That effect can also be seen in our model. However, unlike the conventional model, the resulting $\sigma_{B R}\left(\sigma_{W}\right)$ values from Equation (3) are progressively placed above this line when $\sigma_{W}$ decreases for $\sigma_{W}<1 \mathrm{~S} / \mathrm{m}$. In Figure 7 , one can

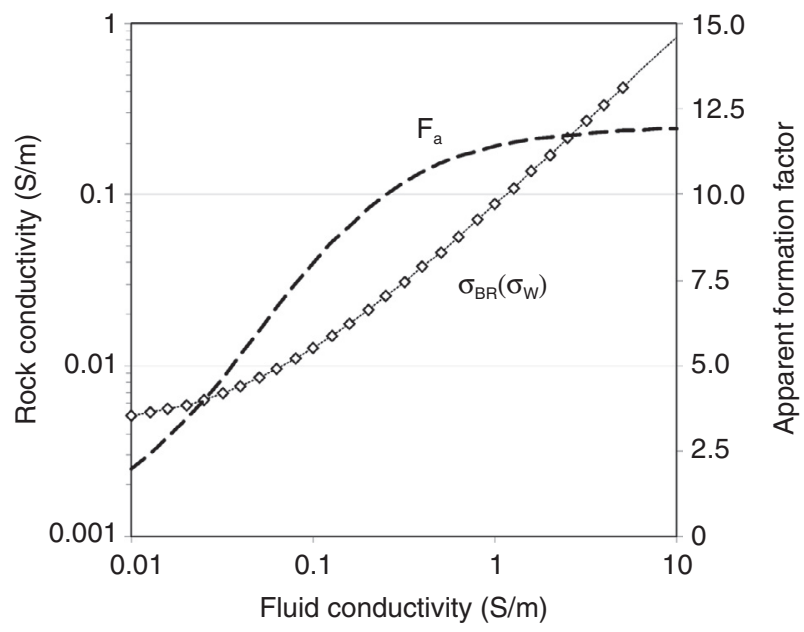

Figure 7

Example of rock conductivity versus pore fluid conductivity and apparent formation factor, $F_{a}=\sigma_{B R} / \sigma_{W}$, according to Equation (3f). see that for very low $\sigma_{W}$ values, the rock conductivity tend to a constant value higher than the trend of $\sigma_{B R}\left(\sigma_{W}\right)$, that is, the conductivity of a rock partially filled with clay, $\sigma_{B R 0}=(V s h / \varnothing) /\left(F \cdot \rho_{C}\right)$ (in this example $\left.\sigma_{B R 0}=0.0042 \mathrm{~S} / \mathrm{m}\right)$, because the remainder is virtually a non-conducting fluid.

Regarding the factors required to obtain $F$ from the clay content (Eq. 3), it is important to point out that, in practice, it is possible to measure both the clay content $\left(V_{s} h\right)$ through the gamma ray value of each layer, and the clay resistivity value $\left(\rho_{C}\right)$ through the measurements in the adjacent layers. Nevertheless, two problems can be found, the presence of non-conductive fluids in clays, and changes in the clay type. In this work, we do not consider the presence of oil and gas in clays, although it is something that should be done in future. About the resistivity changes derived from variations on the type of clay, it seems logical to consider that the type of clay will not change very much in clayey sand layers adjacent to the clay layers. In the same way, it should also be noted that, except for a specific ore, the combinations of clays that appear in most geological formations result in a reduced range of resistivity. In most cases, the clay resistivity value varies from $\sim 5$ to $\sim 15 \Omega \cdot \mathrm{m}$, with a mean value of around $\sim 10 \Omega \cdot \mathrm{m}$.

The main limitation of the established procedure for eliminating the influence of clay on resistivity logs is the hypothesis that it is valid to adopt the same formation factor for clay and for fluid. In our opinion, as for Waxman and Smits (1968), this does not mean that conduction through the clay follows the same pathway as through the formation fluid, or that clay and fluid have the same geometrical factor. What is relevant in this hypothesis is that the opposition to both ionic conductions increases or decreases in the same proportion. However, the hypothesis concerning the relevance of the same geometric factor provides a possible limitation of Equation (3e) for obtaining the formation factor in the presence of clays.

\subsection{About the Influence of Effective Porosity}

In the literature one can find another argument that limits the use of Archie's first law to obtain the appropriate formation factor and is, therefore, a reason why the research of the permeability estimation did not focus on using the formation factor. The porosity used in Archie's first law is fully occupied by free fluid, whereas when there is a "retained" fluid, the part that it occupies, does not contribute to the porosity represented by that equation. In such cases, the part that holds the free fluid is called effective porosity (Archie, 1950; Wyllie and Rose, 1950; Burdine, 1953; Brooks and Corey, 1964). This effective porosity (fraction of total porosity which allows fluid flow under the influence of a pressure gradient) is equivalent to the electrical porosity. Then, it 
should be more appropriate to add the subscript " $E$ " to the porosity in Archie's first law, referred to electrical porosity.

As we have already said, the porosity that satisfies Archie's first law is not the total porosity but the one occupied by "free" water when there is not any other conductive element in the media. Therefore, Archie's first law cannot be used a priori to obtain porosity because the term $\emptyset_{E}{ }^{m}$ involves two unknown factors $\left(\varnothing_{E}\right.$ and “ $m$ "), providing the same value for different combinations of them. This indeterminacy remains today (Thompson et al. 1987; Ahmadi and Quintard, 1996; Mohaghegh et al., 1995; Slater, 2007; Azar et al., 2008).

Fortunately, the result of the developed relationship to obtain the formation factor (Eq. 3e) does not change if it is considered that the fraction of isolated pores filled with fluid is the same than the fraction of isolated pores filled with clay.

Regarding the conversion of $k(F)$ to $k(\varnothing)$, it should be remarked that once the $k(F)$ relationship has been determined by the use of the $F$ values obtained from $\varnothing$ or $\varnothing_{E}$, the transformation to $k(\varnothing)$ can be done through the same process but using a different cementation exponent. In the case where the total porosity is considered, the cementation exponent will be " $m$ ", and if the effective porosity is used, the equivalent cementation exponent " $m_{E}$ " complies with:

$$
1 / \varnothing^{m}=1 / \varnothing_{E}^{m_{E}}
$$

so the resulting permeability values are the same.

It is therefore important to remember that the functions we used satisfy both the $(k, F)$ data and the $(k, \varnothing)$ data from the literature. This was a complex process that required knowledge of different mathematical expressions and testing processes to adjust to all mentioned data (especially its geometry).

\subsection{About the Geometry of $k(\varnothing)$ Functions}

First of all, there have been several possible explanations presented in the literature concerning the different correlations, positive and negative, between porosity and permeability. Alger (1966) stated that this effect, in freshwater environments, is due to a progressive importance of the surface conductivity and to a reduction in the influence of electrolytic conductivity. Purvance and Andricevic (2000) made a similar statement. Independent of the classification and compaction of the grains, we think that this phenomenon is directly understandable from the reduction of grain size, whose effect on permeability is greater than that produced by the increase of porosity.

In this context, we would point out that, in essence, the shape of the obtained $k(\varnothing)$ curves from Equation (5b) are not contrary to Kozeny's expression (Eq. 1b), as may seem at first comparison. The apparent discrepancy is produced when, contrary to what we recommend, a single grain size for all layers of a certain formation is used in Kozeny's equation, because the $\varnothing$ dependence then comes to be $f(\varnothing)=\emptyset^{3} /\left(1-\varnothing^{2}\right)$, and permeability should grow asymptotically to infinity when porosity tends to 1 . However, unconsolidated media have a decreasing grain size with increasing porosity, that is, silts have greater porosity than sands.

In order to make a comparison, let us consider that sedimentary media have an average grain size $d_{a}$ between $0.2 \mathrm{~cm}$ for $25 \%$ porosity and $1 / 256 \mathrm{~cm}$ for porosity values equal to $1 \%$ and $49 \%$. Let us also consider that the grain size shows, for example, a distribution given by the expression $d_{a}=0.2 \cdot \exp \left[-68 \cdot(\varnothing-0.25)^{2}\right]$, which is centred at $25 \%$ porosity. We would note that this distribution provides similar average grain sizes for consolidated media than classical ones. Then, the curve of Kozeny's expression takes a very similar shape to that obtained in this work, as shown in Figure 8. We must mention that the shape of this curve would be very similar for any continuous distribution with the same ranges of grain sizes and porosity.

The main difference between the Kozeny curve, showed in Figure 8, and the obtained results with our model (Fig. 4) is that Equation (5b) provides several curves because it takes into account the effects of sorting and packing of the medium (among other geometrical factors), through the cementation exponent values. Statistically speaking, in sedimentary media the greater the mean grain size the greater permeability and Kozeny's expression come to reflect the $(k, \varnothing)$ values in these media.

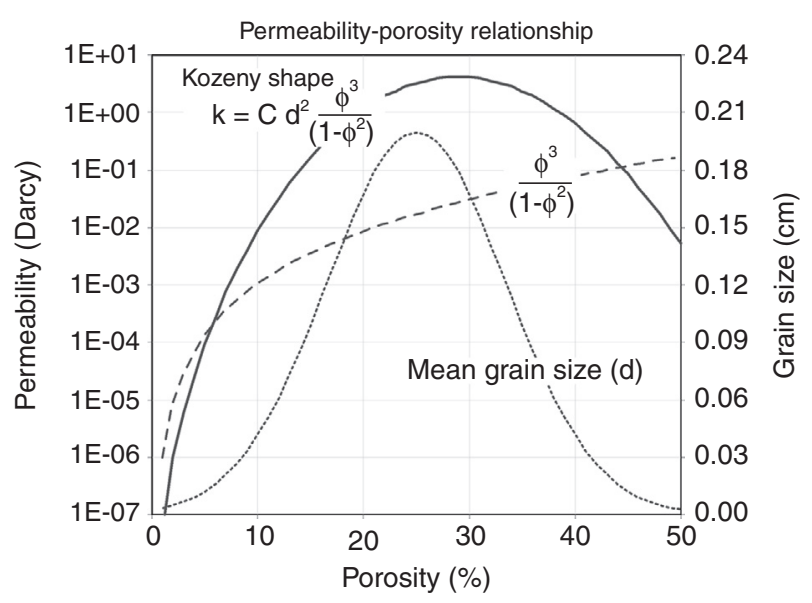

Figure 8

Permeability versus porosity curve according to Kozeny's expression (solid line), for the case of a Gaussian distribution (dotted line) with an average grain size from $2 \mathrm{~mm}$ for porosity of $25 \%$, to $1 / 256 \mathrm{~mm}$ for porosities of $0 \%$ and $50 \%$. Dashed line: function $\varnothing^{3} /\left(1-\varnothing^{2}\right)$. 
At this point, it should be highlighted that there are no works that have obtained permeability functions $k(\varnothing)$ neither in all ranges from 0 up to 50\% porosity, nor showing a similar behaviour to the ones presented in this paper, namely using quasi-symmetric convex curves with different position along the $\varnothing$-axis for different cementation exponents.

However, for consolidated media (porosity lower than $\sim 25 \%$ ) the curves obtained in this work are similar to those obtained with Equation (21) of Jorgensen (1989):

$$
k=\left[C / S_{E}^{2}\right] \cdot \varnothing^{m+2} /[1-\emptyset]^{2}
$$

who included a graph with $k(\varnothing, m)$ curves, Equation (15) of Bayles et al. (1989):

$$
k=1 /\left[8 c \cdot S_{E}^{2}\right] \cdot \varnothing^{2+m} /[1-\varnothing]^{2}
$$

and Equation (A-12) of Glover et al. (2006):

$$
k=\left[3 d_{g r}^{2} / 32\right] \cdot \varnothing^{3 m} / m^{2}
$$

In these works, the permeability was expressed as a function of porosity and geometric coefficients, using the cementation exponent as an added factor. Exclusively for the purpose of illustrating the dependence of these functions with $m$, in Figure 9 we show the results of these equations using the appropriate coefficients (their constants, $S_{E}$, and $d_{g r}$ ) so that for $m=2$ similar permeability values were obtained in the $0-25 \%$ porosity range.

As it can be seen in Figure 9, the works of these authors suggest that the long-established variability of $k(\varnothing)$ relationships lies in a dependence on the cementation exponent. Moreover, if according to the decrease of grain size in the gravel-sand-silt sequence, the variation of $S_{E}$ and $d_{g r}$ were included in the referred equations, the result would be similar to that obtained for unconsolidated media with Equation (5b) for $k(\varnothing)$.

\subsection{Explanatory Examples of $k(\varnothing)$ Behaviour}

Figure 10 shows some examples of $(k, \varnothing)$ pairs of values, in order to explain to what extent the curves are interrelated.

Consider, first, two media with the same permeability, for example, a sandstone with $\varnothing=6 \%$ and $m=1.40$, and another with $\varnothing=15 \%$ and $m=2.10$; the formation factors are close to $F=51.3$ for both media. The most probable reason why these media have the same permeability $\left(9.7 \cdot 10^{-4}\right.$ Darcy) lies in the fact that the latter is more cemented. Similarly, an unconsolidated sand with $\varnothing=38 \%$ and $m=1.20$, has a formation factor of $F=3.19$, which is practically equal to that of a silt with $\varnothing=46 \%$ and $m=1.50$, and both have the same permeability value $\left(2.5 \cdot 10^{-1}\right.$ Darcy). In this case, the most probable reason why these media have the same

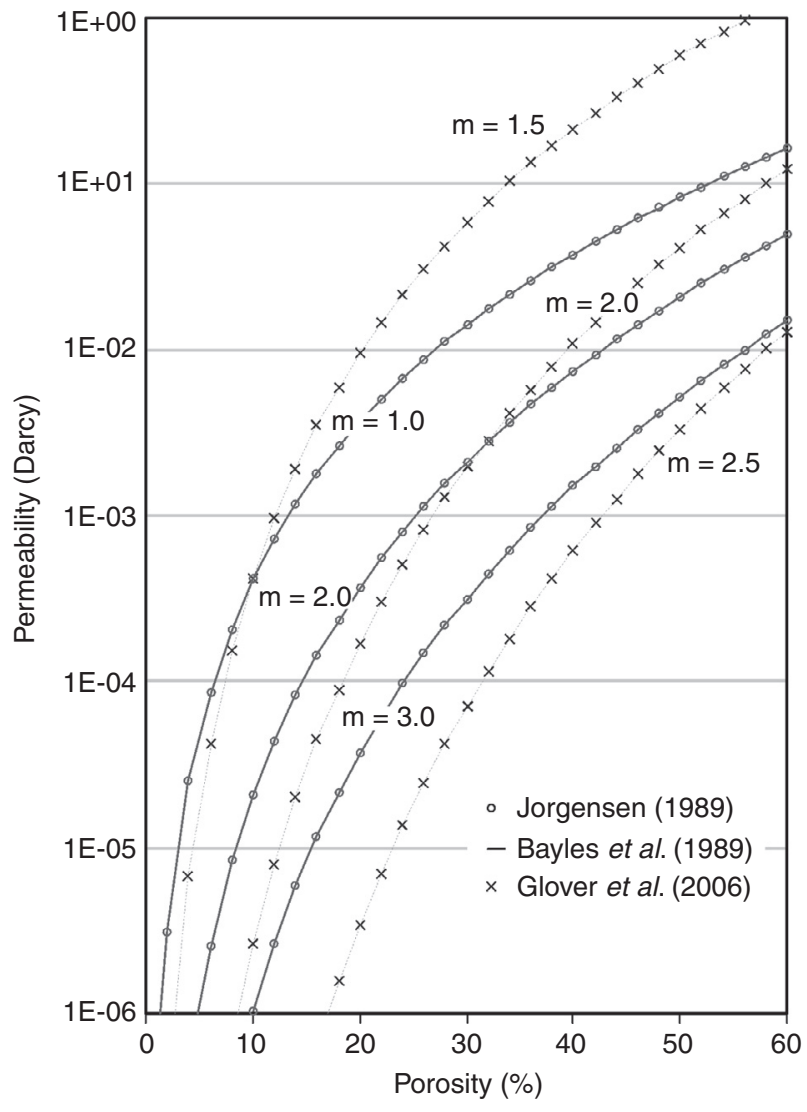

Figure 9

Permeability versus porosity functions with a given pore diameter using the cementation exponent as added factor.

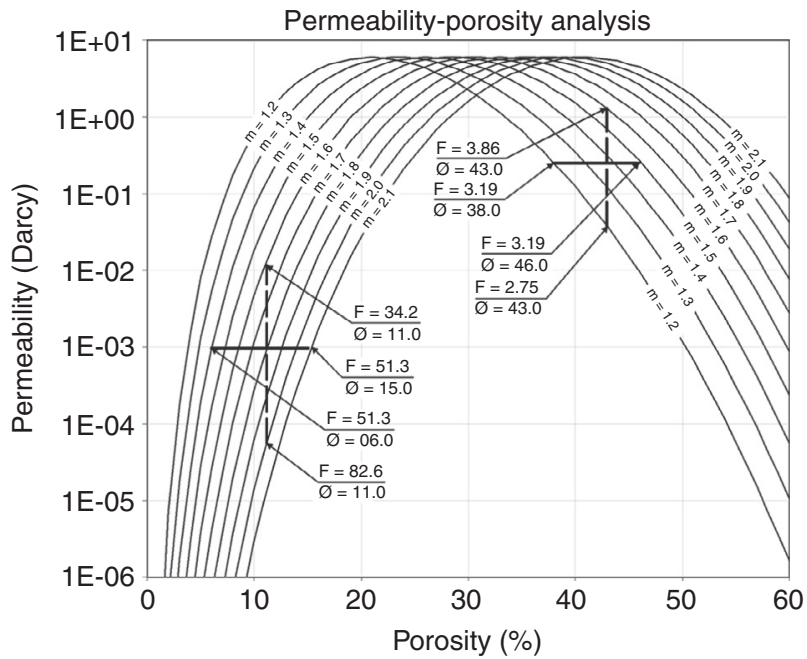

Figure 10

Examples of consolidated and unconsolidated media with different cementation exponent. Horizontal lines: same formation factor and permeability. Vertical lines: same porosity with different formation factor. 
permeability, despite the difference of grain size, is that they present very different sorting.

On the other hand, if we consider different media with the same porosity value, it is noticeable that a high-permeability sandstone, for example with $\varnothing=11 \%$ and $m=1.60$, has much greater permeability $\left(1.2 \cdot 10^{-2}\right.$ Darcy) than a more cemented sandstone with $\varnothing=11 \%$ and $m=2.00$ (permeability $8.3 \cdot 10^{-5}$ Darcy). However, unconsolidated sands with $\varnothing=43 \%$ and $m=1.60$ have more permeability (1.3 Darcy) than sandy silts with $\varnothing=43 \%$ and $m=1.20$ (permeability $3.7 \cdot 10^{-2}$ Darcy). Therefore, for a given porosity in consolidated media, the higher the " $m$ " values, which corresponds to higher formation factor, the lower the permeability values. On the contrary, the opposite applies to unconsolidated media.

The inference to be drawn from these comparisons is that there are many combinations of $(m, \varnothing)$ values that confer the same permeability. With our approaches, for each $F$ value, or for each pair of values $(m, \varnothing)$, only one value of permeability is obtained.

\section{CONCLUSIONS}

With the methodology developed in this paper, it is possible to correct the influence of clay presence on formation factor using Equation (3e):

$$
F=\rho_{B R}\left(\frac{(\phi-V s h) / \phi}{\rho_{W}}+\frac{V s h / \phi}{\rho_{C}}\right)
$$

where $\rho_{B R}, \rho_{W}$ and $\rho_{C}$ represent the resistivities of the rock, interstitial fluid and clay, respectively, $\varnothing$ the porosity and $V s h$ the clay content.

Then using Equation (4b):

$$
k=2.0 \cdot 10^{9}(F-1)^{7} / F^{46}
$$

it is possible to obtain the permeability value (in Darcys).

Next, by means of Archie's first law, one can obtain the cementation exponent either for the total porosity or for the effective porosity (electrical porosity) that provide a unique permeability value using the $k(\varnothing)$ relationship (5b):

$$
k=2.0 \cdot 10^{9} \cdot \phi^{7 m} \cdot\left(1-\phi^{m}\right)^{39}
$$

Regarding the scope of the developed equations, we should note that one of the objectives of this study was to obtain relationships that reflect the $(k, \varnothing)$ statistical behaviour occurring in geological sedimentation processes. In this sense, the obtained equations involve, at the same time, the in-cement transformation of silicates (and other elements present in formations) for porosities below $25 \%$, and an increased presence of clays and marls for porosities above
$25 \%$. It also highlights the generality of the developed approaches, and that the conditions of the used data sets are sufficiently different as they come from very different sources. Moreover, both relationships represented in Equation (4b) between permeability and formation factor, and Equation (5b) between permeability and porosity as a function of the cementation exponent, take into account the existence of a non-effective porosity (non-conductive from an electrical viewpoint).

Concerning Equation (5b), we should note that the permeability value for each porosity value is established as a function of the cementation exponent, which is not a problem if Equation (3d) is used to determine the formation factor as a function of the resistivities and clay content. Thus, each permeability value corresponds to different porosity values as a function of the cementation exponent, which is available from $\varnothing$ and $F$. This exponent will include the internal geometry factors of sedimentary environments, especially the sorting and packing of the grains. We could add that, given the dependence on permeability of the cementation exponent, which does not only depend on cementation, we consider that it would be more adequate to name it "permeability exponent".

Although we have used a large amount of permeability, porosity and formation factor data in order to give greater generality to the developed expressions, this may represent a limitation for two different reasons. On one hand, data from the different works consulted have not always been obtained by direct measurements, but rather indirectly. On the other hand, there are anomalous data corresponding to material with unusual characteristics from a geological point of view, such as for example some media with porosities greater than $50 \%$ or less than $5 \%$, which have permeability values around 0.2 Darcy. In this paper, we have not taken into account many of the data representing these extreme cases. For this reason, we do not rule out that future research may optimize the coefficients determined in this work. In any case, any change in the coefficients of the developed expressions will reinforce our objective to show a $k(\varnothing)$ relationship over the entire range of porosities between 0 and $50 \%$.

Regarding the uncertainties of the expressions developed in this work, the $k(F)$ relationship shows an average difference of 0.7 order of magnitude with respect to data extracted from literature. On the other hand, taking into account the wide range of cases to which the equation for estimating $k(\varnothing)$ is applicable, we have only calculated the uncertainty for two of the cases under study (where the value of $m$ was known) one for consolidated formations and another for unconsolidated ones, resulting in an average value of 0.3 order of magnitude. These values can be considered acceptable since they are similar to those found in the literature. 
Finally, not being necessary to emphasize the importance of knowledge of permeability values for oil and water extraction wells, it is worth emphasizing the added value of the contributions made by the developments presented in this paper. The utility of including permeability in the full range of porosity, from 0 to $50 \%$, lies not only in the difference of the resulting values from traditional relationships (with differentiated curves for consolidated and unconsolidated media), but also in a better characterization of more productive media that are located in the middle area (25 to $35 \%$ porosity). A further advantage is the direct application at any borehole where well logs are available, as resistivity, spontaneous potential and natural gamma that are the most conventional logs.

\section{REFERENCES}

Ahmadi A., Quintard M. (1996) Large-scale properties for two-phase flow in random porous media, Journal of Hydrology 183, 69-99.

Alger R.P. (1966) Interpretation of electric logs in fresh water wells in unconsolidated formations, Society of Professional Well Log Analyst 7th Annual Logging Symposium, Houston Texas, 9-11 May. Archie G.E. (1942) The electrical resistivity logs as an aid in determining some reservoirs characteristics, Transactions of the American Institute of Mining and Metallurgical Engineers 146, 54-62.

Archie G.E. (1950) Introduction to petrophysics of reservoir rocks, Bulletin of the American Association of Petroleum Geologists 34, 943-961.

Azar J.H., Javaherian A., Pishvaie M.R., Nabi-Bidhendi M. (2008) An approach to defining tortuosity and cementation factor in carbonate reservoir rocks, Journal of Petroleum Science and Engineering 60, 125-131.

Baker J.A., Healy H.G., Hackett O.M. (1964) Geology and groundwater conditions in the Wilmington-Reading area, Massachusetts, USGS Water-Supply, paper 1694.

Bayles G.A., Klinzing G.E., Chiang S.-H. (1989) Fractal Mathematics Applied to Flow in Porous Systems, Particle \& Particle Systems Characterization 6, 168-175.

Berg R.R. (1970) Method for determining permeability from reservoir rock properties, Gulf Coast Association of Geologic Society Transaction, Shreveport 20, 303-317.

Bernabé Y., Mok U., Evans B. (2003) Permeability-porosity Relationships in Rocks Subjected to Various Evolution Processes, Pure and Applied Geophysics 160, 937-960.

Brooks R.H., Corey A.T. (1964) Hydraulic Properties of Porous Media, Colorado State University, Hydrological Papers No. 3.

Bourbie T., Zinszner B. (1985) Hydraulic and acoustic properties as a function of porosity in Fontainebleau sandstone, Journal of Geophysical Research 90, 11524-11532.

Burdine N.T. (1953) Relative permeability calculations from pore size distribution data, Transactions of American Institute of Mining, Metallurgical and Petroleum Engineers 198, 71-77.

Byrnes A.P., Cluff R.M., Webb J.C. (2009) Analysis of Critical Permeability, Capillary Pressure and Electrical Properties for Mesaverde Tight Gas Sandstones from Western U.S. Basins, Final Scientificl Technical Report DE-FC26-05NT42660 Submitted by University of Kansas Center for Research, Inc. for U.S. Department of Energy.
Carman P.C. (1937) Fluid flow through granular beds, Transactions of Institute of Chemical Engineering 50, 150-166.

Carothers J.E. (1968) A statistical study of the formation factor, The Log Analyst 9, 5, 13-20.

Coates G.R., Dumanoir J.L. (1974) A new approach to improved log derived permeability, The Log Analyst 15, 1, 17-31.

Croft M.G. (1971) A method of calculating permeability from electric logs, USGS Professional, Paper 750-B, pp. B265-B269.

De Lima O.A.L., Sharma M.M. (1990) A grain conductivity approach to shaly sandstones, Geophysics 55, 10, 1347-1356.

De Lima O.A.L., Clennell M.B., Nery G.G., Niwas S. (2005) Volumetric approach for the resistivity response of freshwater shaly sandstone, Geophysics 70, 1-10.

De Witte L. (1950) Relations between resistivities and fluid contents of porous rocks, Oil and Gas Journal 49, 16, 120-132.

Detmer D.M. (1995) Permeability, porosity, and grain-size distribution of selected Pliocene and Quaternary sediments in the Albuquerque Basin, New Mexico Geology 17, 4, 79-87.

Díaz-Curiel J. (1995) Interpretación y correlación automáticas de diagrafías geofísicas. Aplicación a la hidrogeología en el Sur de la cuenca del río Duero, $P h D$ Thesis, Universidad Politécnica de Madrid.

Doll H.G. (1949) The SP Log: Theoretical Analysis and Principles of Interpretation, Transactions of the American Institute of Mining and Metallurgical Engineers 179, 146-185.

Ehrenberg S.N., Eberli G.P., Keramati M., Moallemi A. (2006) Porosity-permeability relationships in interlayered limestonedolostone reservoirs, American Association of Petroleum Geologists Bulletin 90, 1, 91-114.

Ehrlich R., Etris E.L., Brumfield D., Yuan L.P., Crabtreeet S.J. (1991) Petrography and Reservoir Physics III, Physical Models for Permeability and Formation Factor, American Association of Petroleum Geologists Bulletin 75, 10, 1579-1592.

Frohlich R.K., Fisher J.J., Summerly E. (1996) Electric-hydraulic conductivity correlation in fractured crystalline bedrock: Central Landfill, Rhode Island, USA, Journal of Applied Geophysics 35, 249-259.

Glover P.W.J., Zadjali I.I., Frew K.A. (2006) Permeability prediction from MICP and NMR data using an electrokinetic approach, Geophysics 71, 4, F49-F60.

Glover P.W.J., Walker E. (2009) Grain-size to effective pore-size transformation derived from electrokinetic theory, Geophysics $\mathbf{7 4}$, 1, E17-E29.

Heigold P.C., Gilkeson R.H., Cartwright K., Reed P.C. (1979) Aquifer transmissivity from surficial electrical methods, Ground Water 17, 338-345.

Herrick D.C., Kennedy W.D. (1995) Formation resistivity factor and permeability relationships in rocks characterized by secondary solution porosity, 36th SPWLA annual logging symposium, transactions, Paris (France), 26-29 June.

Hill H.J., Milburn J.D. (1956) Effect of clay and water salinity on electrochemical behavior of reservoir rocks, Transactions of the American Institute of Mining and Metallurgical Engineers 207, 65-72.

Huntley D. (1986) Relations between Permeability and Electrical Resistivity in Granular Aquifers, Ground Water 24, 4, 466-474.

Jackson P.D., Taylor D., Stanford P.N. (1978) Resistivity-porosityparticle shape relationships for marine sands, Geophysics 43, 6, 1250-1268. 
Jin M., Sharma M.M. (1994) Shaly sand formation evaluation using a single membrane potential measurement, Journal of Petroleum Science and Engineering 11, 301-310.

Jones P.H., Buford T.B. (1951) Electric logging applied to groundwater exploration, Geophysics 16, 1, 115-139.

Jorgensen D.G. (1989) Using Geophysical Logs to Estimate Porosity, Water Resistivity, and Intrinsic Permeability, U.S. Geological Survey Water-Supply, paper 2321.

Katz A.J., Thompson A.H. (1985) Fractal sandstone pores: implications for conductivity and pore formation, Physical Review Letters 54, 1325-1328.

Kelly W.E., Frohlich R.K. (1985) Relations between Aquifer Electrical and Hydraulic Properties, Ground Water 23, 2, 182-189.

Khalil M.A., Santos F.A.M. (2009) Influence of Degree of Saturation in the Electric Resistivity - Hydraulic Conductivity Relationship, Surveys in Geophysics 30, 601-615.

Kosinski W.K., Kelly E.W. (1981) Geoelectrical sounding for predicting aquifer properties, Ground Water 19, 163-171.

Kozeny J. (1927) Uber die kapillare leitung des wassers im bodenaufstieg versickerung und anwendung auf die bewässerung: Sitzungsberichte der Wiener Akademie der Wissenschaften (Abt. IIa), Math.-Naturwiss, 136, 271-306.

Kwader T. (1985) Estimating Aquifer Permeability from Formation Resistivity Factors, Ground Water 23, 6, 762-766.

Lee M.W. (2011) Connectivity Equation and Shaly-Sand Correction for Electrical Resistivity, USGS Scientific Investigations Report 2011-5005, 9 p.

Mohaghegh S., Balan B., Ameri S. (1995) State-of-the-Art in Permeability Determination from Well Log Data Part II - Verifiable, Accurate Permeability Predictions, the Touch-Stone of All Models, Society of Petroleum Engineers, SPE 30979.

Morrow N.R., Huppler J.D., Simmons A.B. (1969) Porosity and permeability of unconsolidated upper miocene sands from grain size analysis, Journal of Sedimentary Petrology 39, 312-321.

Mounce W.D., Rust W.M. (1944) Natural Potentials in Well Logging, Transactions of the American Institute of Mining and Metallurgical Engineers 155, 49-57.

Nelson P.H. (1994) Permeability-Porosity Relationships in sedimentary rocks, The Log Analyst 35, 38-62.

Ogbe D., Bassiouni Z. (1978) Estimation of aquifer permeabilities from electric well logs, The Log Analyst 19, 5, 21-27.

Pape H.G., Clauser C.H., Iffland J. (1999) Permeability prediction based on fractal pore-space geometry, Geophysics 64, 5, 1447-1460.

Paterson M.S. (1983) The equivalent channel model for permeability and resistivity in fluid saturated rocks - A reappraisal, Mechanics of Materials 2, 4, 345-352.

Patnode W.H., Wyllie M.R.J. (1950) The presence of conductive solids in reservoir rocks as factor in electric log interpretation, Petroleum Transactions of the American Institute of Mining and Metallurgical Engineers 189, 47-52.

Porter C.R., Carothers J.W. (1970) Formation factor - porosity relationship derived from well log data, Society of Professional Well Log Analys 11th Annual Logging Symposium, Los Angeles, California, 3-6 May.

Purvance D.T., Andricevic R. (2000) On the electrical-hydraulic conductivity correlation in aquifers, Water Resources Research 36, 2905-2913.

Revil A., Cathles L.M. (1999) Permeability of Shaly Sands, Water Resources Research 35, 651-662.
Salazar J.M., Wang G.L., Torres-Verdín C., Lee H.J. (2008) Combined simulation and inversion of SP and resistivity logs for the estimation of connate-water resistivity and Archie's cementation exponent, Geophysics 73, 3, E107-E114.

Salem H.S., Chilingarian G.V. (1999) The cementation factor of Archie's equation for shaly sandstone reservoirs, Journal of Petroleum Science and Engineering 23, 83-93.

Sawyer W.K., Pierce C.I., Lowe R.B. (1971) Electrical and hydraulic flow properties of Appalachian petroleum reservoir rocks, 10th Annual Conference Ontario Petroleum Institute Inc., Toronto, Canada, 24-25 Oct.

Schopper J.R. (1966) A theoretical investigation on the formation factor-permeability-porosity relationship using a network model, 28th meeting of the EAGE, Amsterdam, June. Reprinted in Geophysical Prospecting 14, 3, 301-341.

Sen P.N., Straley C., Kenyon W.E., Whittingham M.S. (1990) Surface to volume ratio, charge density, nuclear magnetic relaxation and permeability in clay-bearing sandstones, Geophysics 55, 1, 61-69.

Schlumberger C., Schlumberger M., Leonardon E.G. (1934) A New Contribution to Subsurface Studies by Means of Electrical Measurements in Drill Knits, Transactions of the American Institute of Mining and Metallurgical Engineers 110, 273-289.

Slater L. (2007) Near Surface Electrical Characterization of Hydraulic Conductivity; From Petrophysical Properties to Aquifer Geometries - A review, Surveys in Geophysics 28, 169-197.

Slichter S.C. (1899) Theoretical investigation of the motion of ground water, USGS 19th Annual Report, part II, pp. 301-380.

Sundberg K. (1932) Effect of impregnating waters on electrical conductivity of soils and rocks, Transactions of American Institute of Mining and Metallurgical Engineers 97, 367-391.

Thompson A.H., Katz A.J., Krohn C.E. (1987) The microgeometry and transport properties of sedimentary rock, Advances in Physics 36, 625-694.

Timur A. (1968) An investigation of permeability, porosity, and residual water saturation relationships for sandstone reservoirs, Society of Professional Well Log Analyst 9th Annual Well Logging Symposium, New Orleans, 23-26 June.

Turner J.B. (1983) Problematic petrophysical characteristic of the smackover at Bayou Middle Fork field, Clairborne Parish, Louisiana, Gulf Coast Association of Geological Societies Transactions 33, 221-230.

Uren L.C. (1925) Increasing production of petroleum by increasing diameters of wells, Transactions of the American Institute of Mining and Metallurgical Engineers 71, 1276-1300, (New York Meeting, February).

Urish D.W. (1981) Electrical resistivity - hydraulic conductivity relationships in glacial outwash aquifers, Water Resources Research 17, 5, 1401-1408.

van Baaren J.P. (1979) Quick-look permeability estimates using sidewall samples and porosity logs, Transactions on 6th Society of Professional Well Log Analyst Annual European Logging Symposium, London, 26-27 March.

Viberti D., Verga F. (2011) An Approach for the Reliable Evaluation of the Uncertainties Associated to Petrophysical Properties, Mathematical Geosciences 44, 3, 327-341.

Waxman M.H., Smits L.J.M. (1968) Electrical conductivities in oilbearing shaly sands, Society of Petroleum Engineers Journal 243, 107-122. 
Winsauer W.O., Shearin H., Masson P., Williams M. (1952) Resistivity of brine-saturated sands in relation to pore geometry, American Association of Petroleum Geologists Bulletin 2, 253-277.

Winsauer W.O., McCardell W.M. (1953) Ionic double-layer conductivity in reservoir rock, Petroleum Transactions of the American Institute of Mining and Metallurgical Engineers 198, 129-134.

Wong P.-Z., Koplik J., Tomanic J.P. (1984) Conductivity and permeability of rocks, Physical Review B 30, 11, 6606-6614.

Worthington P.F. (1977) Influence of matrix conduction upon hydrogeophysical relationships in arenaceous aquifers, Water Resources Research 13, 87-92.

Worthington P.F. (1983) The relationship of electrical resistivity to intergranular permeability in reservoir rocks, 24th Society of Professional Well Log Analyst Annual Logging Symposium, Calgary, 27-30 June.
Wyllie M.R.J. (1949) A Quantitative Analysis of the Electro Chemical Component of the SP Curve, Journal of Petroleum Technology 1, 1, 17-26.

Wyllie M.R.J., Rose W.D. (1950) Some theoretical considerations related to the quantitative evaluation of the physical characteristics of reservoir rock from electrical log data, Transactions of the American Institute of Mining and Metallurgical Engineers 189, 105-118.

Wyllie M.R.J., Gregory G.R. (1953) Formation factors of unconsolidated porous media: Influence of particle shape and effects of cementation, Petroleum Transactions of American Institute of Mining and Metallurgical Engineers 198, 103-110.

Manuscript submitted in July 2014 Manuscript accepted in November 2014

Published online in March 2015

Cite this article as: J. Díaz-Curiel, B. Biosca and M.J. Miguel (2016). Geophysical Estimation of Permeability in Sedimentary Media with Porosities from 0 to 50\%, Oil Gas Sci. Technol 71, 27. 